\title{
Premature pulmonary valve opening in a patient with absent tricuspid valve
}

\author{
IVAN A D'CRUZ, SHIRISH N SHAH, RICHARD LEVINSKY, \\ ALBERTO GOLDBARG
}

From the Cardiovascular Institute, Department of Medicine, Michael Reese Hospital and Medical Center, and the University of Chicago Pritzker School of Medicine, Chicago, Illinois, USA

SUMMARY Echocardiography ( $M$-mode and cross-sectional) disclosed premature opening of the pulmonary valve (very deep " $a$ " wave) in a young man who underwent tricuspid valvulectomy without valve replacement 32 months before for refractory bacterial endocarditis. Cardiac catheterisation showed a prominent atrial wave on the pulmonary artery pressure curve. Thus, part of the right ventricular ejection appeared to occur before the onset of ventricular systole, as a result of powerful right atrial contraction.

Unusually large " $a$ " waves on the pulmonary valve echo, with failure of the pulmonary cusp to return to its closed position before the onset of ventricular systole, have been described as characteristic of moderate to severe pulmonary valve stenosis. ${ }^{1}$ In a young man who had had surgical removal (but not replacement) of his tricuspid valve 32 months previously for bacterial endocardititis which was refractory to medical treatment, $M$-mode as well as cross-sectional echocardiography disclosed premature opening of the pulmonary valve. A prominent presystolic " $a$ " wave was noted on the pulmonary artery pressure curve.

\section{Case report}

A 23-year-old heroin addict presented with high fever in September 1976 caused by bacterial endocarditis (Streptococcus viridans) affecting the tricuspid valve. In spite of intensive chemotherapy, he became progressively more febrile and toxic; serial chest $x$-ray showed multiple areas of infarction thought to be caused by successive pulmonary embolisation and echocardiography disclosed large tricuspid valve vegetations. In view of the failure of medical treatment, open heart surgery to excise the infected tricuspid valve was performed; prosthetic valve replacement was not done. The patient made an uneventful recovery and has been living a normal unrestricted life since then, with no symptoms and no limitation of effort tolerance.
On examination (May 1979) prominent jugular venous pulsations and hyperdynamic right ventricular parasternal pulsations were noted. The first heart sound was audible at the apex but not at the left sternal border. No murmur was heard. There was no evidence of congestive heart failure. Right atrial and ventricular enlargement were evident on teleradiography of the chest. The electrocardiogram showed right ventricular hypertrophy.

On M-mode echocardiography (Picker System $80 \mathrm{C}$ ), the right ventricle appeared dilated and the ventricular septum showed paradoxical (anterior) systolic motion, attributable to right ventricular diastolic overloading. A very large " $a$ " wave was seen on the pulmonary valve echo (Fig. 1) and the cusp did not return to its closed position before the beginning of ventricular systole. Crosssectional echocardiography, viewing the aortic root and pulmonary artery in short-axis view, confirmed that the pulmonary valve opened before the onset of the QRS complex (Fig. 2).

Right heart catheterisation was performed the day after the echocardiogram was recorded. The right atrial pressure curve was typical of severe tricuspid regurgitation $(a=16, v=20 \mathrm{~mm}$, mean $=15 \mathrm{~mm})$. The pulmonary artery pressure was normal (peak systolic $=22 \mathrm{~mm}$, mean $=17 \mathrm{~mm}$ ), but the pressure tracing showed a prominent "a" wave $(18 \mathrm{~mm})$ which followed the onset of the $P$ wave of the electrocardiogram, but preceded the onset of the QRS complex (Fig. 3).

The cardiac output was $6.01 / \mathrm{min}$ and the cardiac 
index $2.7 \mathrm{l} / \mathrm{min}$ per $\mathrm{m}^{2}$ (Fick method). Angiocardiography (right ventricular injection) showed severe tricuspid regurgitation and dilatation of the right atrium and ventricle.

\section{Comment}

Very large "a" waves on the pulmonary valve echo, sometimes referred to as premature pulmonary

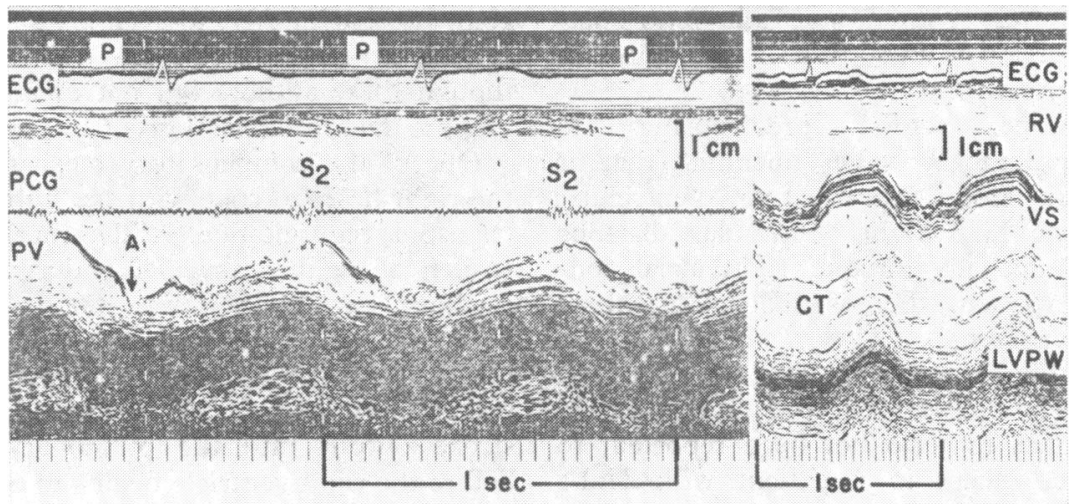

Fig. $1 M$-mode echocardiogram. In the left panel, the pulmonary valve $(P V)$ is seen to open fully after the onset of the $P$ wave (large " $a$ " wave), well before the onset of the QRS. ECG, electrocardiogram; PCG, phonocardiogram; $S_{2}$, second heart sound. The right panel shows the left ventricle at chordae tendineae (CT) level. The right ventricle $(R V)$ is dilated and the ventricular septum (VS) shows paradoxical anterior systolic motion. LVPW, left ventricular posterior wall.

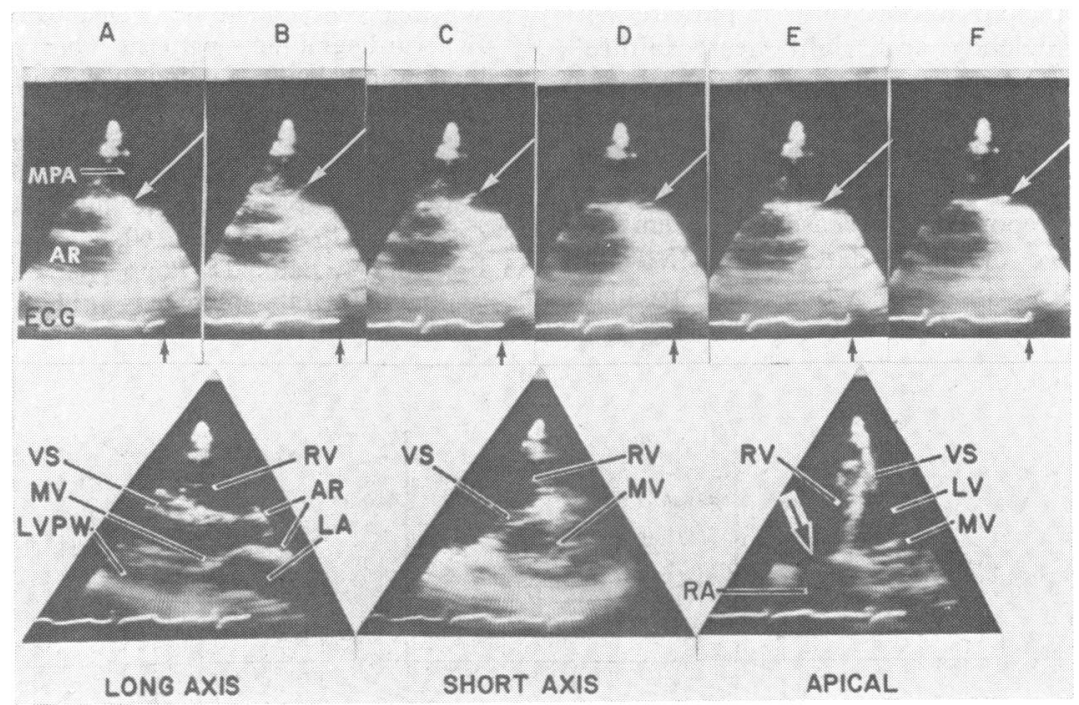

Fig. 2 Cross-sectional echocardiogram. The upper row of six frames shows the posterior cusp of the pulmonary valve in different phases of the cardiac cycle, which are indicated on the electrocardiogram by the small black upward arrows. The cusp is in an open position in systole (frame A), then closes in early to mid-diastole (B and C), but opens in late diastole (D and $\mathrm{E}$ ) after onset of the $P$ wave. Frame $\mathrm{F}$, during inscription of the $S$ wave, approximates onset of ventricular systole. AR, aortic root; $M P A$, main pulmonary artery. The lower three frames, in the long axis, short axis, and apical views, respectively, were all taken during systole. They show that the right ventricle ( $R V)$ is dilated, while the left ventricle $(L V)$ is of normal size. The arrow in the apical view indicates where the tricuspid valve would have been seen if it was present. VS, ventricular septum; MV, mitral valve; LVPW, left ventricular posterior wall; LA, left atrium; $R A$, right atrium. 
valve opening, are considered an important sign of moderate to severe pulmonary valve stenosis. ${ }^{1}$ This finding has also been noted in a patient with aorta-right atrial fistula resulting from rupture of a sinus of Valsalva aneurysm, ${ }^{2}$ in a patient with Uhl's anomaly of the right ventricle ${ }^{3}$ in a patient with pulmonary regurgitation and right ventricular outflow obstruction, ${ }^{4}$ and also occasionally in constructive pericarditis. ${ }^{5}$

Haemodynamic factors predisposing to "atriogenic" pulmonary valve opening (that is valve opening before the onset of ventricular systole), include high right ventricular diastolic pressure and unusually forceful right atrial contraction. In our patient, there is evidence that these two factors are present.

The presystolic prominent " $a$ " wave on the pulmonary artery pressure curve is an interesting feature which has hitherto not been reported, to our knowledge, except in a patient with Uhl's anomaly. ${ }^{3}$ It is attributable to unusually strong contraction of the right atrium, which in turn may result from diastolic overload of this chamber (Starling's law). Chronic massive tricuspid regurgitation, in our patient, could have produced atrial hypertrophy.

Feigenbaum ${ }^{5}$ mentioned having "noted premature opening of the pulmonic valve in patients with tricuspid insufficiency and right heart failure", but added "the pulmonic valve presumably opens following rapid rise in right ventricular pressure in early diastole that occurs in these conditions". In another report from Feigenbaum's laboratory, Wann et al. ${ }^{6}$ reported five cases of premature pulmonary valve opening, detected by $\mathrm{M}$-mode echocardiography, three of whom had tricuspid regurgitation. The authors concluded that, in all their patients, "alterations in right ventricular diastolic pressure relative to pulmonary artery diastolic pressure account for opening of the pulmonary valve without the contribution of atrial or ventricular systole".

That surgical removal of the tricuspid valve is compatible with life and even the absence of severe cardiac symptoms has been well documented, ${ }^{78}$ though these authors did not include echocardiographic data in their reports.

One of the compensatory mechanisms enabling the right heart to cope with the burden of massive tricuspid regurgitation is the increased diastolic stretch of right ventricular and right atrial myocardium (Starling's law). Another mechanism, demonstrated in our case, is the ability of the right atrium to shoulder part of the right heart diastolic overload by ejecting part of the right heart stroke output into the pulmonary artery, even before the right ventricle begins to contract.

This patient was operated on by Dr C E Anagnostopoulos.

\section{References}

1 Weyman AE, Dillon JC, Feigenbaum H, Chang S. Echocardiographic patterns of pulmonic valve motion in valvular pulmonary stenosis. Am $\mathcal{F}$ Cardiol 1974; 34: 644-51.

2 Weyman AE, Dillon JC, Feigenbaum H, Chang S. Premature pulmonic valve opening following sinus of Valsalva aneurysm rupture into the right atrium. Circulation 1975; 51: 556-60.

3 French JW, Baum D, Popp RL. Echocardiographic findings in Uhl's anomaly. Am $\mathcal{F}$ Cardiol 1975; 36: $349-53$.

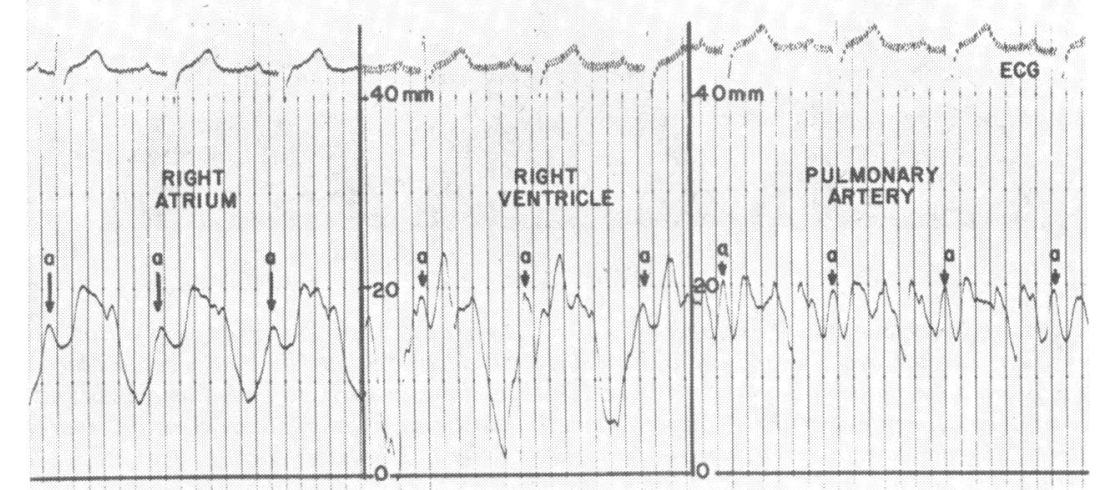

Fig. 3 Pressure tracings obtained during right heart catheterisation. The right atrial and right ventricular pressure curves are similar and show a large regurgitant " $v$ " wave, preceded by a prominent " $a$ " wave. Note the unusual finding of an " $a$ " wave on the pulmonary artery pressure curve. 
4 Weyman AE, Dillon JC, Feigenbaum H, Chang S. Pulmonary valve echo motion in pulmonary regurgitation. Br Heart f 1975; 37: 1184-90.

5 Feigenbaum H. Echocardiography. Philadelphia: Lea \& Febiger, 1976.

6 Wann LS, Weyman AE, Dillon JC, Feigenbaum H. Premature pulmonary valve opening. Circulation 1977; 55: 128-33.

7 Robin E, Thoms NW, Arbulu A, Ganguly SN, Magnisalis K. Hemodynamic consequences of total removal of the tricuspid valve without prosthetic replacement. Am f Cardiol 1975; 35: 581-6.

8 Sethia B, Williams BT. Tricuspid valve excision without replacement in a case of endocarditis secondary to drug abuse. Br Heart f 1978; 40: 579-80.

Requests for reprints to Dr Ivan D'Cruz, Cardiovascular Institute, Michael Reese Hospital and Medical Center, 29th Street \& Ellis Avenue, Chicago, Illinois 60616, USA. 